\title{
Which hormonal management approach for women with premature ovarian insufficiency is best for bone?
}

\section{The use of combined oral contraceptives (COCs) in 119 women with a mean age of 30.3 years who had premature ovarian insufficiency was associated with the most positive trends in bone mineral density (BMD). Bone density scans} revealed that women who used COC or high-dose estrogen plus progesterone therapy (EPT) had increases in BMD at the lumbar spine, while women who used no treatment or low-dose EPT experienced declines in lumbar spine BMD.

Carvalho Gazarra LB, Bonacordi CL, Yela DA, et al. Bone mass in women with premature ovarian insufficiency: $a$ comparative study between hormone therapy and combined oral contraceptives. Menopause. 2020;27:1110-1116.

\section{EXPERT COMMENTARY}

Andrew M. Kaunitz, MD, is Professor and Associate Chairman, Department of Obstetrics and Gynecology, University of Florida College of Medicine-Jacksonville; Medical Director and Director of Menopause and Gynecologic Ultrasound Services, UF Women's Health Specialists at Emerson, Jacksonville. He serves on the OBG MANAGEMENT Board of Editors.

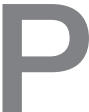

remature ovarian insufficiency (POI) refers to a condition in women in whom ovarian function ceases prior to age 40 years. Although hormone therapy (HT) is a mainstay of treatment for women with POI, it is uncertain which approach to HT is most effective in terms of bone mineral

The author reports serving on the advisory boards of Pfizer (contraception) and Mithra, and that the University of Florida has received clinical trial support from Mithra.

doi: 10.12788/obgm.0048 density (BMD). Investigators recently published their results of an observational study that aimed to evaluate the use of combined oral contraceptives (COCs) for preserving BMD in women with POI.

\section{Details of the study}

At an academic center in Brazil, Carvalho Gazarra and colleagues identified women with POI who had undergone 2 or more BMD assessments performed 2 or more years apart. ${ }^{1}$ HT regimens (all of which were taken continuously) employed the following: a COC with ethinyl estradiol (EE) $30 \mu \mathrm{g}$ and levonorgestrel; low-dose estrogen plus progestin therapy (EPT, conjugated equine estrogen [CEE] $0.625 \mathrm{mg}$ with medroxyprogesterone acetate or estradiol $1.0 \mathrm{mg}$ with norethindrone acetate); or high-dose estrogen plus progestin (CEE $1.25 \mathrm{mg}$ or estradiol $2.0 \mathrm{mg}$ combined with the same progestins).

Results. Among 119 evaluable women with POI (mean age, 30.3 years), the use of COC was associated with the most positive BMD trends. For women using COC or high-dose EPT, BMD at the lumbar spine increased. By contrast, BMD of the lumbar spine declined
FAST

TRACK

Although HT is a mainstay of treatment for women with $\mathrm{POI}$, it is uncertain which approach to HT is most effective in terms of bone mineral density 


\section{WHAT THIS EVIDENCE MEANS FOR PRACTICE}

When replacing estrogen and progestin in young women who lack ovarian function, it is appropriate to use considerably higher doses than those used to treat bothersome vasomotor symptoms in women with normal/spontaneous menopause. From the perspective of venous thromboembolism risk, the transdermal route of administration is safer than the oral route, ${ }^{5}$ and the Scottish and US studies discussed here indicate that transdermal estradiol is an effective approach to maintaining skeletal health in young women without ovarian function. Accordingly, hormonal management with high-dose transdermal estradiol with a progestin (such as progesterone 200-300 mg at bedtime or medroxyprogesterone 5-10 mg daily) represents an appropriate strategy. In situations where transdermal estradiol plus oral progestin treatment is not covered by health insurance or acceptable to the patient, an oral estrogen-progestin contraceptive formulated with EE 30 or $35 \mu \mathrm{g}$ will provide protection against bone loss.

in women who used no treatment or lowdose EPT.

\section{Other studies' take on dose, route of administration, and cost considerations}

Sequelae of POI include infertility, bothersome hot flashes, vaginal dryness, sexual dysfunction, mood disorders, and an elevated risk of cardiovascular disease, dementia, Parkinson's disease, and osteoporosis. Importantly, clinicians and patients need to understand that the results from the Women's Health Initiative studies do not apply to women with POI. ${ }^{2}$ Physiologic doses of HT (that is, doses higher than those used to treat menopausal symptoms in women with normal/spontaneous menopause) are appropriate for women with POI, at least until they reach the normal age of menopause (51 to 52 years).

A clinical trial conducted in Scotland in women with POI found that high-dose transdermal estrogen (application of one to two 0.1-mg estradiol patches) daily had an impact on BMD that was more positive than that of an oral contraceptive formulated with EE $30 \mu \mathrm{g} .{ }^{3}$ Likewise, a trial in the United States found that, among oligo-amenorrheic athletes, a hormone replacement regimen using a 0.1-mg estradiol patch had a more positive impact on BMD than an oral contraceptive formulated with EE $30 \mu \mathrm{g}{ }^{4}$

Although Carvalho Gazarra and colleagues acknowledged awareness of reports suggesting the skeletal health benefits of high-dose estradiol patches, in the Brazilian public health system oral hormone therapy is less expensive and oral contraceptives are available at no charge. ${ }^{1}$
References

1. Carvalho Gazarra LB, Bonacordi CL, Yela DA, et al. Bone mass in women with premature ovarian insufficiency: a comparative study between hormone therapy and combined oral contraceptives. Menopause. 2020;27: 1110-1116.

2. Jiang XD. Bone health and beyond in women with primary ovarian insufficiency: time to narrow the knowledge-action gap in care. Menopause. 2020;27:1101-1103.

3. Crofton PM, Evans N, Bath LE, et al. Physiological versus standard sex steroid replacement in young women with premature ovarian failure: effects on bone mass acquisition and turnover. Clin Endocrinol (Oxf). 2010;73:707-714.

4. Ackerman KE, Singhal V, Baskaran C, et al. Oestrogen replacement improves bone mineral density in oligoamenorrhoeic athletes: a randomised clinical trial. Br J Sports Med. 2019;53:229-236.

5. Vinogradova Y, Coupland C, Hippisley-Cox J. Use of hormone replacement therapy and risk of venous thromboembolism: nested case-control studies using the QResearch and CPRD databases. BMJ. 2019;364:k4810. 